\title{
Collisional Transport in Axisymmetric Plasma Columns with Strong Longitudinal Flows: Application to Solar Loops
}

\author{
V. S. Tsypin ${ }^{a)}$ and R.M.O. Galvão ${ }^{a), b}$ \\ a) Institute of Physics, University of São Paulo, Cidade Universitária, CEP 05508-900, São Paulo, Brazil and \\ b) Brazilian Center for Research in Physics, Rua Xavier Sigaud, 150, CEP 22290-180, Rio de Janeiro, Brazil
}

Received on 7 January, 2005

\begin{abstract}
In this work we analyze the transport processes in solar loops considering a collisional plasma and high longitudinal plasma flow. The general theory of the neoclassical transport in toroidal configurations with a noncircular cross-section is applied to explain the transport processes in some kinds of solar loops, modeling the solar loop as a toroidal plasma column. The plasma is assumed to be in the collisional regime and to have particle longitudinal flow along the solar loop axis. The poloidal velocity and the radial fluxes of the particles and the ion heat flux are derived in this article. It is shown that the particle poloidal velocity can be measured giving rise to the possibility of having additional connections between the plasma macroscopic parameters, which are very important for the solar loops diagnostic. It is also shown that the particle and heat fluxes are on the "classical" level, within the factor of an order of magnitude. We hope that such an approach (together with other theories) can help to explain the transport processes in solar loops, whose shapes are similar to toroidal configurations.
\end{abstract}

\section{INTRODUCTION}

Solar loops are usual structures on the surface of stars. There are extensive investigations of the different physical processes in solar loops, both, observational and theoretical [1]. The transport processes in the solar loops are relatively poorly studied because of their complex structure and the necessity of taking into account a large amount of physical processes present in them.

The solar loops have different shapes, indeed some of them just rise above the surface of the sun while others look like the toroidal configurations of fusion devices (Priest 1980, page 378) [2]. Fortunately, the theory of plasma dynamics in toroidal configurations has been rather well developed in connection with the research of thermonuclear fusion. Specifically, it is supposed that the ion transport is neoclassical (i.e. the classical collisional transport [3]), taking into account additionally the complicated topology of magnetic field) in all collisional regimes. Indeed, the neoclassical theory consistently includes the collisional regime, when the mean free path is small in comparison with the typical longitudinal size of the system, the weakly collisional one (the "plateau" regime, when the transport coefficients do not depend on the collision frequency), and the "banana" regime, when it is necessary to take into account the so-called trapped particles, [4]. The electron dynamics in a collisional regime can be also described in the framework of the neoclassical theory. A comprehensive review of the neoclassical transport theory has been published by Hinton and Hazeltine [5].

The plasma of solar loops, in some cases, is collisional with high longitudinal particle flow velocities, not far from the plasma sound speed. The ratio of the plasma pressure $p_{0}$ to the magnetic field pressure $B^{2} / 8 \pi$ is usually small, i.e. the parameter $\beta_{p}=8 \pi p_{0} / B^{2}$ is much smaller than one. The toroidal (along the solar loop) magnetic field $B_{\zeta_{p}}$ is usually greater than the poloidal magnetic field $B_{\theta p}, \quad B_{\zeta_{p}} \gg B_{\theta p}$. Under these conditions, we can neglect the radial gradients of the magnetic field in the equations describing the plasma dynamics. The solar loops transverse cross-section can differ from a circular one and the temperature is high enough for the plasma to be considered as fully ionized [6].

Here, we want to construct the transport processes theory valid for solar loops as in toroidal magnetically confined collisional two-component (ions and electrons) plasma configurations, with high longitudinal (along the torus) particle flow velocities and elongated (elliptical) transverse crosssection. Previously, such a problem was solved for axisymmetric plasma columns with circular cross-section, in cases in a plateau [7], collisional [8], [9], and all transport regimes [10].

Of course, there are fundamental differences between the solar loops and tokamaks. The first difference is the sun strong gravitational field. The second is the vertical position of the many loops. The third is the absence of strong inductive currents in the loops, as in the case for tokamaks. Finally, the feet of solar loops is on the surface of the Sun. These differences need special considerations. We confine ourselves to consider only the local characteristics of the plasma dynamics, such as the local plasma transport. The sun gravitational field affects primarily the plasma longitudinal flow. Simplifying the problem, we average the evolution equations only over the azimuthal angle, at the same coordinate along the loop. In some sense, we replace the flow velocity, depending on the longitudinal coordinate, by the mean flow velocity. In this case, as a first approximation, we neglect the dependence of the macroscopic plasma parameters on the longitudinal coordinate, along the torus. There is a similarity between tokamaks with the poloidal divertor, where the magnetic field lines are unclosed, and the solar case with the feet of the loops tied to the solar surface. Furthermore, the longitudinal transport dominates the energy and particles balance in many kinds of solar loops, however, for interpretation of the observational data, it is necessary to evaluate the particle and energy amount crossing the solar loop surface. In some kinds of solar loops (such as shown in the book by Priest, page 378 [2]), the transverse transport can dominate the lon- 
gitudinal one because of the solar loop large outer surface, in comparison with the transversal area of the feet.

We are aware that, even for strongly toroidal solar loops, we do not take into account all possible ranges of variation of the plasma parameters. In some kinds of solar loops, the effects of radial gradients, curvature of the magnetic field, and the derivatives of the plasma parameters along the torus can be of the same order of the effects considered here. These problems can be the subject of future investigations. In this paper, we confine ourselves to consider only the comparatively simple case of solar loops with the complicated geometry of the magnetic field.

The model equilibrium magnetic field and the associated coordinate system and metric are described in section 2 to 4 . The particle and heat fluxes are derived in sections 5 and 6, respectively. The ambipolarity condition is discussed in section 7. Using this condition, the final expressions for the transport fluxes are obtained and evaluated, for some cases of interest, in sections 8 and 9. Finally, the conclusions are presented in section 10 .

\section{MAGNETIC FIELD}

First of all, we need to choose the coordinate system convenient to evaluate the heat and particle fluxes in the elliptical plasma torus. For a smooth torus (no dependence of the plasma macroscopic parameters on the coordinate along the toroidal column), it is convenient to take two coordinates in a transverse cross-section of a plasma column (a radial coordinate and a azimuthal, poloidal, coordinate) and an angle coordinate along torus. The coordinates should be chosen so that the dependence of the plasma macroscopic parameters on them should be as simple as possible. For example, the plasma macroscopic parameters should depend mainly on the radial coordinate and be approximately constant along the magnetic surfaces for a torus with a small inverse aspect ratio (the ratio between the small and large radii of the plasma column). It can be shown, from the approximate equilibrium equation [3], that

$$
\frac{1}{c}[\mathbf{j} \times \mathbf{B}] \approx \nabla p,
$$

where $\mathbf{B}$ is the magnetic field, $\mathbf{j}$ is the plasma current, and $p$ is the full plasma isotropic pressure. Multiplying this equation by $\mathbf{B}$, we have

$$
\mathbf{B} \cdot \nabla p=0,
$$

i.e. the plasma pressure is approximately constant along the magnetic field line and on the magnetic surface (naturally, this condition should be valid on the magnetic surface close to the feet of the solar loop). Thus, it is convenient to choose the radial coordinate so that it is constant on a magnetic surface. Possible candidates for this coordinate are the plasma volume inside the magnetic surface $V$, the toroidal $\phi$ or poloidal $\chi$ magnetic fluxes, and any functions of these values. If a radial coordinate does not coincide with the magnetic surface, strong angle dependence will appear in dynamic equations and the calculations will be highly complicated. In a toroidal magnetic configuration with elliptic transverse cross-section, it is possible to take a function of semi minor and major axes as the radial coordinate, as will be done in the sequel.

Let us designate the poloidal and toroidal coordinates (angles) $\theta$ and $\zeta$ respectively, where $0 \leq \theta \leq 2 \pi$ and $0 \leq \zeta \leq 2 \pi$. Using the Maxwell equation

$$
\nabla \cdot \mathbf{B}=0
$$

we obtain the identities

$$
\nabla \cdot(\mathbf{B} \theta)=\mathbf{B} \cdot \nabla \theta, \quad \nabla \cdot(\mathbf{B} \zeta)=\mathbf{B} \cdot \nabla \zeta .
$$

Let us make the cutting of torus in any place across the plasma column and denote the meaning of the angle $\zeta$ as $\zeta_{1}=0$ on the one side of the cutting and $\zeta_{2}=2 \pi$ on the other side of the cutting. Then, integrating the second equation of Eqs. (4) over the plasma volume inside a given magnetic surface of the obtained magnetic configuration, using the absence of the radial component of the magnetic field on the surface, and the conservation of the toroidal magnetic flux inside it, we get the expression for the toroidal magnetic flux (see, for example, [11])

$$
\phi=\frac{1}{2 \pi} \int(\mathbf{B} \cdot \nabla) \zeta d \mathbf{r} .
$$

Analogously, let us make the cutting of torus in any place along the plasma column from its center until the magnetic surface and denote the meaning of the angle $\theta$ as $\theta_{1}=0$ on the one side of the cutting and $\theta_{2}=2 \pi$ on the other side of the cutting. Then, integrating the first equation of Eqs. (4) over the plasma volume inside the surface and using the conservation of the poloidal magnetic flux, we get the expression for the quantity

$$
\chi=\frac{1}{2 \pi} \int(\mathbf{B} \cdot \nabla) \theta d \mathbf{r}
$$

Now, we can define the magnetic field $\mathbf{B}\left(B^{r}=0\right)$

$$
\mathbf{B}=\left\{0 ; \quad B^{\theta} ; \quad B^{\zeta}\right\}
$$

where $B^{\theta}=(\mathbf{B} \cdot \nabla) \theta$ and $B^{\zeta}=(\mathbf{B} \cdot \nabla) \zeta$ are the contravariant poloidal and toroidal components of the magnetic field, respectively. From Eq. (3), allowing for Eq. (7), we obtain

$$
\frac{1}{\sqrt{g}} \frac{\partial}{\partial \theta}\left(\sqrt{g} B^{\theta}\right)+\frac{1}{\sqrt{g}} \frac{\partial}{\partial \zeta}\left(\sqrt{g} B^{\zeta}\right)=0
$$

where $g$ is the metric tensor determinant (see below). This equation can be satisfied if we take (Shafranov \& Soloviev 1969) [11]

$$
B^{\theta}=-\frac{1}{2 \pi \sqrt{g}} \frac{\partial H(r, \theta, \zeta)}{\partial \zeta}, \quad B^{\zeta}=\frac{1}{2 \pi \sqrt{g}} \frac{\partial H(r, \theta, \zeta)}{\partial \theta}
$$

( $r$ is some radial coordinate) and

$$
H(r, \theta, \zeta)=A_{1}(r) \theta+A_{2}(r) \zeta+\tilde{H}(r, \theta, \zeta),
$$


where $\tilde{H}(r, \theta, \zeta)$ is some periodic function of the angles $\theta$ and $\zeta$, so as $B^{\theta}$ and $B^{\zeta}$. Using Eqs. (5), (6), and (10) and calculating the incremental toroidal and poloidal magnetic fluxes between neighbor magnetic surfaces $r=$ const and $r+d r=$ const , we find

$$
\begin{gathered}
d \phi=\frac{1}{2 \pi} \int(\mathbf{B} \cdot \nabla) \zeta d \mathbf{r}=A_{1}(r) d r \\
d \chi=\frac{1}{2 \pi} \int(\mathbf{B} \cdot \nabla) \theta d \mathbf{r}=-A_{2}(r) d r
\end{gathered}
$$

and

$$
A_{1}(r)=\phi^{\prime}, \quad A_{2}(r)=-\chi^{\prime}
$$

where "," designates the radial derivative. As a result, we have

$$
\mathbf{B}=\left\{B^{r} ; \quad B^{\theta} ; \quad B^{\zeta}\right\}=\left\{0 ; \quad \frac{\chi^{\prime}}{2 \pi \sqrt{g}} ; \quad \frac{\phi^{\prime}}{2 \pi \sqrt{g}}\right\} .
$$

The definition of the safety factor $q$, which plays an important role in a plasma stability theory, is

$$
q=\frac{B^{\zeta}}{B^{\theta}}=\frac{\phi^{\prime}}{\chi^{\prime}}
$$

in any coordinate system. We see from this equation that $q$ depends only on the radial coordinate $r$, i.e. in the chosen coordinate system, the magnetic field lines are straight; the magnetic field lines do not depend on the poloidal and toroidal angles on a magnetic surface, what simplifies significantly calculations.

Now, we can find the metric and the coordinate dependence of macroscopic plasma values in the coordinate system with straight magnetic field lines.

\section{COORDINATE SYSTEM}

At first, we take the orthogonal coordinates $\rho^{\prime}, \omega^{\prime}$ and $\zeta$ (where $\rho^{\prime}$ is the "radial" coordinate, $\omega^{\prime}$ and $\zeta$ are the poloidal and toroidal angles respectively) to be attached to some points on the magnetic surfaces and to the geometric center of their transverse cross-section.

The length element in this orthogonal coordinate system is

$$
d l^{2}=d \rho^{\prime 2}+\rho^{\prime 2} d \omega^{\prime 2}+\left(R-\rho^{\prime} \cos \omega^{\prime}\right)^{2} d \zeta^{2} .
$$

Here, $R$ is the torus major radius. The next step is the encircling of the magnetic surfaces by means of the transformation

$\rho \cos \omega=\exp (\eta / 2) \rho^{\prime} \cos \omega^{\prime}, \quad \rho \sin \omega=\exp (-\eta / 2) \rho^{\prime} \sin \omega^{\prime}$,

where $\rho=\sqrt{l_{1} l_{2}}$ is the new "radial" coordinate, $\eta=\ln \left(l_{2} / l_{1}\right)$, $l_{1}$ and $l_{2}$ are the semi minor and major axes of the torus elliptical cross-section, respectively, $\omega$ is the new poloidal angle. We suppose also that for all magnetic surfaces the ratio $l_{2} / l_{1}$ is the same. Although, as it will be shown below, the ellipticity usually does not give a substantial contributions to the final expressions for the transport fluxes, we believe that it is useful to take the ellipticity into account methodically for future investigations in this direction, for cases, where the ellipticity may be substantial. Then, we focus on the geometric center of the solar loop cross-section and straighten the magnetic line of force by means of small variations of the poloidal angle with the help of the parameter $\delta(\theta, r)$, introduced by the expressions

$$
\begin{gathered}
\rho \cos \omega=r \cos [\theta+\delta(\theta, r)]+\Delta(r), \\
\rho \sin \omega=r \sin [\theta+\delta(\theta, r)],
\end{gathered}
$$

where $\theta$ is the final poloidal angle, $r$ is the final radial coordinate, $\Delta(r)$ is the Shafranov displacement of the geometric center of the magnetic surfaces, when the plasma cylinder is transformed into torus, see below.

We find the straightening parameter $\delta(\theta, r)$, from the condition of vanishing radial currents $\left(j^{r}=0\right)$, taking into account the expression for the magnetic field $\mathbf{B}$, with the force straight line Eq. (14), supposing the torus to be axisymmetric $(\partial / \partial \zeta=0)$. We make this approximation as the first step to solve the problem under investigation. This approximation is valid provided that $r \ll R$, as in this case, for example,

$$
\frac{\partial p}{\partial r} \gg \frac{1}{R} \frac{\partial p}{\partial \zeta}
$$

and we can omit terms with the longitudinal derivatives in the plasma dynamic equations. Of course, this model needs further investigation, taking into account the longitudinal (along torus) derivatives of macroscopic values in the dynamic equations.

\section{METRIC}

To find the metric tensor components $g_{i k}$ and their determinant, we substitute Eqs. (17), (18) into Eq. (16) to obtain

$$
d l^{2}=g_{i k} d x^{i} d x^{k}=g_{11} d r^{2}+2 g_{12} d r d \theta+g_{22} d \theta^{2}+g_{33} d \zeta^{2},
$$

where $x^{1}=r, x^{2}=\theta, x^{3}=\zeta$. Then, from the contravariant radial component of the Maxwell equation (neglecting the displacement current and assuming $j^{r}=0$ ), we have

$$
\nabla \times \mathbf{B}=\frac{4 \pi}{c} \mathbf{j}
$$

namely

$$
\frac{\partial B_{\zeta}}{\partial \theta}=0, \quad B_{\zeta}=g_{33} B^{\zeta}
$$

where $B_{\zeta}$ is the $\zeta$-covariant component of the magnetic field. Using Eq. (14) we find that

$$
\frac{\partial}{\partial \theta}\left(\frac{g_{33}}{\sqrt{g}}\right)=0,
$$


where, within a $\varepsilon^{* 2}$ accuracy, it follows from Eqs. (16)-(19) that

$$
\sqrt{g}=r R\left(1-\varepsilon^{*} \cos \theta-\Delta^{\prime} \cos \theta+\frac{\partial \delta}{\partial \theta}\right)
$$

and

$$
g_{33}=R^{2}\left(1-2 \varepsilon^{*} \cos \theta\right) .
$$

As a result, we derive from Eqs. (22)-(24) that the parameter $\delta$ is equal to

$$
\delta=-\sin \theta\left[\varepsilon^{*}+\Delta^{\prime} \exp (\eta / 2)\right]
$$

and the metric tensor components and their determinant become

$$
g_{11}=\cosh \eta-\sinh \eta \cos 2 \theta+2 \Delta^{\prime} \exp (-\eta / 2) \cos \theta,
$$

$$
\begin{gathered}
g_{22}=r^{2}(\cosh \eta+\sinh \eta \cos 2 \theta) \\
{\left[1-2 \cos \theta\left(\varepsilon^{*}+\Delta^{\prime} \exp (\eta / 2)\right)\right],} \\
g_{12}=g_{21}=r\left\{\sinh \eta \sin 2 \theta-\sin \theta\left[\operatorname { c o s h } \eta \left(\varepsilon^{*}+\right.\right.\right. \\
\left.\left.\left.+r \Delta^{\prime \prime} \exp (\eta / 2)\right)+\Delta^{\prime} \exp (-\eta / 2)\right]\right\}, \\
g_{33}=R^{2}\left(1-2 \varepsilon^{*} \cos \theta\right), \quad \sqrt{g}=r R\left(1-2 \varepsilon^{*} \cos \theta\right),
\end{gathered}
$$

where the parameter $\varepsilon^{*}$ is $\varepsilon^{*}=\varepsilon \exp (-\eta / 2), \varepsilon=r / R \ll 1$, $\varepsilon^{*} \ll 1$.

Now, using Eqs. (14), (26) - (29), we can find the angular dependence of the magnetic field

$$
B=\sqrt{g_{i k} B^{i} B^{k}},
$$

which is

$$
B=B_{s}\left(1+\varepsilon^{*} \cos \theta+\frac{A \varepsilon^{*}}{2} \cos ^{2} \theta\right),
$$

where

$$
A=\frac{\varepsilon^{*}[\exp (2 \eta)-1]}{q^{2}}, \quad B_{s}=\frac{\phi^{\prime}}{2 \pi r} .
$$

We shall also need a component of the Cristoffel's symbol

$$
\Gamma_{k l}^{i}=g^{i m} \Gamma_{m, k l}, \quad \Gamma_{m, k l}=\frac{1}{2}\left(\frac{\partial g_{m k}}{\partial x^{l}}+\frac{\partial g_{m l}}{\partial x^{k}}-\frac{\partial g_{k l}}{\partial x^{m}}\right),
$$

namely,

$$
\Gamma_{\zeta \theta}^{\zeta}=\varepsilon^{*} \sin \theta
$$

where $x^{i}=(r, \theta, \zeta)$
The Shafranov displacement parameter $\Delta$ can be found from Eq. (1) (the equilibrium equation). In the zero approximation to the parameter $\varepsilon^{*}$, we have the usual equilibrium equation

$$
\frac{d}{d r}\left(8 \pi p+B^{2}\right)=-\frac{2 B_{\theta p}^{2}}{r},
$$

where $B_{\theta p}$ is the physical $\theta$ - component of the magnetic field. In the next approximation on the parameter $\varepsilon^{*}$, we have

$$
\frac{d}{d r}\left[r B_{\theta p}^{2}\left(\Delta^{\prime}+\varepsilon^{*}\right)-r \varepsilon^{*} B_{\theta p}^{2}\right]=\varepsilon^{*}\left(8 \pi r \frac{d p}{d r}-B_{\theta p}^{2}\right) .
$$

To find the parameter $\Delta$, it is necessary to know the radial distribution of the plasma pressure and current. In laboratory toroidal devices, they usually have the parabolic radial dependence.

\section{PARTICLE TRANSPORT}

The particle fluxes $\Gamma_{n}$ can be found by integrating the electron continuity equation

$$
\frac{\partial n}{\partial t}=-\nabla \cdot\left(n \mathbf{V}_{e}\right)
$$

over the plasma volume, enclosed by a given magnetic surface, and using the Gauss theorem

$$
\Gamma_{n}=<n V_{e}^{r}>
$$

where $n$ is the plasma density, $\mathbf{V}_{e}=\mathbf{V}_{e \perp}+V_{e \|} \mathbf{h}$ is the electron macroscopic velocity and

$$
<\ldots>=\int_{0}^{2 \pi}(\ldots) \sqrt{g} d \theta d \zeta / \int_{0}^{2 \pi} \sqrt{g} d \theta d \zeta .
$$

We find the contravariant radial component of the electron velocity using the electron motion equation [3]

$$
M_{e} n \frac{d_{e} \mathbf{V}_{e}}{d t}=-\nabla p_{e}-\nabla \cdot \hat{\pi}_{e}+e_{e} n\left(\mathbf{E}+\frac{1}{c}\left[\mathbf{V}_{e} \times \mathbf{B}\right]\right)+\mathbf{R}_{e}
$$

where $M_{e}, p_{e}, \hat{\pi}_{e}$ are the electron mass, pressure, and viscosity, respectively, and

$$
\frac{d_{e}}{d t}=\frac{\partial}{\partial t}+\mathbf{V}_{e} \cdot \nabla, \quad \mathbf{R}_{e}=\mathbf{R}_{u}+\mathbf{R}_{T} .
$$

The electron-ion friction force is equal to

$$
\mathbf{R}_{u}=-e_{e} n\left(\frac{\mathbf{j}_{\perp}}{\sigma_{\perp}}+\frac{\mathbf{j}_{\|}}{\sigma_{\|}}\right)
$$

where

$$
\mathbf{j}_{\perp}=[\mathbf{h} \times[\mathbf{j} \times \mathbf{h}]], \quad \mathbf{j}_{\|}=\mathbf{h}(\mathbf{h} \cdot \mathbf{j})
$$

are the plasma transverse and longitudinal currents,

$$
\sigma_{\perp}=\frac{e_{e}^{2} n \tau_{e}}{M_{e}}, \quad \sigma_{\|}=1.96 \sigma_{\perp}
$$


are the plasma transverse and longitudinal electrical conductivity, respectively, and the thermal force term is given by the expression [3]

$$
\mathbf{R}_{T}=-0.71 n \nabla_{\|} T_{e}+\frac{3}{2} \frac{n T_{e}}{\omega_{B e} \tau_{e}}\left[\mathbf{h} \times \nabla T_{e}\right], \quad \mathbf{h}=\frac{\mathbf{B}}{B} .
$$

Here, $\omega_{B e}=e_{e} B / M_{e} c$ is the electron cyclotron frequency, $\tau_{e}$ is the electron-ion collision characteristic time, and $T_{e}$ is the electron temperature.

The transverse current is found by summing the ion and electron motion equations

$$
\mathbf{j}_{\perp}=\frac{c}{B_{0}}\left[\mathbf{h} \times\left(\nabla p+\nabla \cdot \pi+M_{i} n \frac{d_{i} \mathbf{V}_{i}}{d t}\right)\right],
$$

where we have neglected the electron inertia.

Let us find the $\zeta$ - covariant component of Eqs. (38) and (39), neglecting the small terms of $\varepsilon^{* 2}$ and assuming that $\partial / \partial \zeta=0$

$$
\begin{gathered}
\frac{e_{e} \sqrt{g}}{c} V_{e}^{r} B^{\theta}-\frac{e_{e}}{\sigma_{\perp}}\left(\mathbf{j}_{\perp}\right)_{\zeta}-\frac{e_{e}}{\sigma_{\|}}\left(\mathbf{j}_{\|}\right)_{\zeta} \\
-\frac{3}{2} \frac{\sqrt{g} T_{e}}{\omega_{B e} \tau_{e}} \frac{B^{\theta}}{B}\left(\nabla T_{e}\right)^{r}-0.71\left(\nabla_{\|} T_{e}\right)_{\zeta}=0 .
\end{gathered}
$$

From Eq. (41), we have

$$
\left(\mathbf{j}_{\perp}\right)_{\zeta}=\frac{c \sqrt{g} B^{\theta}}{B^{2}}(\nabla p)^{r}=\frac{c \sqrt{g} B^{\theta} g^{11}}{B^{2}} \frac{\partial p}{\partial r}
$$

and then

$$
\begin{gathered}
\left(\mathbf{j}_{\|}\right)_{\zeta}=\left(j_{\|} \mathbf{h}\right)_{\zeta}=\frac{j_{\|} g_{33} B^{\zeta}}{B}, \\
\left(\nabla_{\|} T_{e}\right)_{\zeta}=\left[\mathbf{h}(\mathbf{h} \cdot \nabla) T_{e}\right]_{\zeta}=\frac{g_{33} B^{\zeta} B^{\theta}}{B} \frac{\partial T_{e}}{\partial \theta},
\end{gathered}
$$

where, as it follows from Eqs. (26) - (28),

$$
g^{11}=\frac{g_{22}}{g_{11} g_{22}-g_{12}^{2}}=\cosh \eta+\sinh \eta \cos 2 \theta+\varepsilon^{*} \sinh \eta \cos \theta \text {. }
$$

Using Eqs. (42) - (45), we obtain

$$
V_{e}^{r}=-\frac{c^{2} g^{11}}{\sigma_{\perp} B_{s}^{2}}\left(\frac{\partial p}{\partial r}-\frac{3}{2} n \frac{\partial T_{e}}{\partial r}\right)+\frac{c q j_{\|}}{\sigma_{\|} \varepsilon B_{s}}+\frac{0.71 c}{e_{e} r B_{s}} \frac{\partial T_{e}}{\partial \theta} .
$$

Here we have omitted terms which disappear after the surface averaging, Eq. (37), for example, terms of the kind $\sin \theta \cos \theta$.

In Eq. (47), the first term is the usual classical one. The second term [12] and the thermal force term [13] are neoclassical, taking into account the complicated topology of magnetic field, depending on the poloidal angle $\theta$. The oscillating with the angle $\theta$ longitudinal current $j_{\|}$can be derived from the current continuity equation $\nabla \cdot \mathbf{j}=0$

$$
h^{\theta} \frac{\partial j_{\|}}{\partial \theta}=-\nabla \cdot \mathbf{j}_{\perp}
$$

as $\mathbf{j}=\mathbf{j}_{\perp}+\mathbf{j}_{\|}, \mathbf{j}_{\|}=\mathbf{h} j_{\|}$, and

$$
\nabla \cdot \mathbf{j}_{\|}=\frac{1}{\sqrt{g}} \frac{\partial}{\partial \theta}\left(\frac{\sqrt{g} j_{\|} B^{\theta}}{B}\right) \approx h^{\theta} \frac{\partial j_{\|}}{\partial \theta},
$$

$h^{\theta}$ is the $\theta$-contravariant component of the unit vector $\mathbf{h}$. We have taken into account the plasma quasineutrality $\left(n_{e} \approx n_{i} \approx\right.$ $n$ ), when we obtained the current continuity Eq. (48), summing the electron and ion continuity equations (Eq. (36)).

As it follows from Eq. (41), we have

$$
\nabla \cdot \frac{c}{B}\left[\mathbf{h} \times M_{i} n \frac{d_{i} \mathbf{V}_{i}}{d t}\right] \approx-\frac{c M_{i}}{\sqrt{g}} \frac{\partial}{\partial r}\left[\frac{g_{33} B^{\zeta} n\left(\left(\mathbf{V}_{i} \cdot \nabla\right) \mathbf{V}_{i}\right)_{\theta}}{B^{2}}\right]
$$

and from Eq. (33), $\left(V_{\|}^{2} \approx g_{22} V_{\theta}^{2}+g_{33} V_{\zeta}^{2} \approx V_{\zeta} V^{\zeta}\right)$

$$
\left(\left(\mathbf{V}_{i} \cdot \nabla\right) \mathbf{V}_{i}\right)_{\theta} \approx V^{\zeta} \nabla_{\zeta} V_{\theta} \approx-V_{\zeta} V^{\zeta} \Gamma_{\zeta \theta}^{\zeta} \approx-V_{\|}^{2} \varepsilon^{*} \sin \theta,
$$

as $V^{\theta} \ll V^{\zeta}$, and

$$
\nabla_{\zeta} V_{\theta}=\frac{\partial V_{\theta}}{\partial \zeta}-V_{k} \Gamma_{\zeta \theta}^{k}
$$

Using Eqs. (41), (48) - (51), we obtain

$$
\frac{\partial j_{\|}}{\partial \theta}=\frac{c q g_{33}}{B \sqrt{g}} \frac{\partial p}{\partial r} \frac{\partial \ln B}{\partial \theta}-\frac{c h^{\zeta}}{\varepsilon B} \frac{\partial}{\partial r}\left(M_{i} n V_{\|}^{2} \varepsilon^{*}\right) \sin \theta,
$$

where Eq. (14) was used, and $V_{\|}$is the plasma longitudinal flow velocity. Using Eq. (14), we obtain

$$
\begin{aligned}
j_{\|} & =\frac{c q}{B_{s}} \frac{\varepsilon^{*}}{\varepsilon}\left[2\left(\cos \theta+\frac{A}{4} \cos 2 \theta\right) \frac{\partial p}{\partial r}\right. \\
& \left.+\alpha p\left(\frac{1}{r}+\kappa_{n}+2 \kappa_{V}\right) \cos \theta\right],
\end{aligned}
$$

where

$$
\kappa_{V}=\frac{\partial \ln V_{\|}}{\partial r}, \quad \alpha=\frac{V_{\|}^{2}}{c_{s}^{2}}, \quad c_{s}^{2}=\frac{T_{i}+T_{e}}{M_{i}} .
$$

For a circular plasma $(A=0)$ and if the parameter $\alpha$ is neglected, the expression for $j_{\|}$, Eq. (53), reduces is to the wellknown Pfirsch and Schlüter current [12].

To derive an expression for the term $\partial T_{e} / \partial \theta$, we should proceed from the longitudinal heat flux expression [3]

$$
q_{\| e}=-\frac{3.16 \sigma_{\perp} T_{e}}{e_{e}^{2} q R} \frac{\partial T_{e}}{\partial \theta}+\frac{0.71 T_{e}}{e_{e}} j_{\|} .
$$

The longitudinal heat flux can be found from the temperature evolution equation [3]. In our case it is given by

$$
h^{\theta} \frac{\partial q_{\| e}}{\partial \theta}=-\nabla \cdot \mathbf{q}_{\perp e}+p_{e} V_{e}^{\theta} \frac{\partial n}{\partial \theta}
$$


where $V_{e}^{\theta}$ is the contravariant poloidal component of the electron macroscopic velocity and

$$
\mathbf{q}_{\perp e}=\frac{5}{2} \frac{p_{e}}{\omega_{B e} M_{e}}\left[\mathbf{h} \times \nabla T_{e}\right] .
$$

Thus, in the case of small $\beta_{p}$, we can omit the radial derivatives of the magnetic field $B$. This is possible to do, as for nonoscillating terms with the angle $\theta$ under condition $B_{\theta p} \ll B$ we obtain from Eq. (34)

$$
\kappa_{B} \approx \frac{\beta_{p}}{2} \kappa_{p}, \quad \kappa_{B}=\frac{\partial \ln B_{s}}{\partial r}, \quad \kappa_{p}=\frac{\partial \ln p}{\partial r} .
$$

For terms that oscillate with the angle $\theta$, the radial derivative of the magnetic field, Eq. (31), gives terms that vanish with the surface average and we obtain

$$
\nabla \cdot \mathbf{q}_{\perp e}=\frac{5}{2} \frac{p_{e} U_{T e}}{r} \frac{\partial}{\partial \theta} \ln \frac{n}{B^{2}}, \quad U_{T e}=\frac{1}{M_{e} \omega_{B e}} \frac{\partial T_{e}}{\partial r} .
$$

We can now write down the expression for the particle fluxes, Eq. (37)

$$
\begin{gathered}
\Gamma_{n e}=-\frac{c^{2} n \cosh \eta}{\sigma_{\perp} B^{2}}\left(\frac{\partial p}{\partial r}-\frac{3}{2} n \frac{\partial T_{e}}{\partial r}\right)+ \\
+\frac{1}{2 \pi r R} \int_{0}^{2 \pi} d \theta\left(\frac{n c q \sqrt{g} j_{\|}}{\varepsilon \sigma_{\|} B_{s}}+\frac{0.71 c n \sqrt{g}}{r e_{e} B_{s}} \frac{\partial T_{e}}{\partial \theta}\right),
\end{gathered}
$$

valid up to order $\varepsilon^{* 2}$. From Eqs.(47), (54) - (57), we see that to derive the particle fluxes we need to find the poloidal dependence of the particle density, $n=n(\theta)$, and the poloidal particle velocity $V_{j}^{\theta}$. This is, by itself, a separate problem, that should be solved by taking into account the ambipolarity particle fluxes, as will be done in section 7 .

\section{ION HEAT FLUX}

As in the previous part, we derive the ion heat flux, $\Gamma_{T i}$, integrating the temperature evolution equation [3]

$$
\frac{3}{2} n \frac{d_{i} T_{i}}{d t}+p_{i} \nabla \cdot \mathbf{V}_{\mathbf{i}}=-\nabla \cdot \mathbf{q}_{\mathbf{i}}
$$

over the plasma volume

$$
\Gamma_{T i}=<q_{i}^{r}>.
$$

In Eq. (59) we neglect an ion viscosity and an ion-electron heat exchange. The heat flux radial contravariant component is

$$
q_{i}^{r}=-\frac{2 p_{i} v_{i} g^{11}}{M_{i} \omega_{B i}^{2}} \frac{\partial T_{i}}{\partial r}-\frac{5}{2} \frac{p_{i} g_{33} h^{\zeta}}{M_{i} \omega_{B i} \sqrt{g}} \frac{\partial T_{i}}{\partial \theta} .
$$

The second term in this expression is neoclassical [14]. Eq. (61) follows from the expression [3]

$$
\mathbf{q}_{i \perp}=-\frac{2 p_{i}}{M_{i} \omega_{B i}^{2} \tau_{i}} \nabla_{\perp} T_{i}+\frac{5}{2} \frac{p_{i}}{M_{i} \omega_{B i}}\left[\mathbf{h} \times \nabla T_{i}\right] .
$$

The poloidal dependence of the ion temperature can be found from Eqs. (59), allowing for the continuity equation, Eq. (36),

$$
h^{\theta} \frac{\partial q_{\| i}}{\partial \theta}=-\nabla \cdot \mathbf{q}_{\perp i}+p_{i} V_{i}^{\theta} \frac{\partial n}{\partial \theta}
$$

and

$$
q_{\| i}=-\frac{3.9 p_{i}}{v_{i} M_{i} q R} \frac{\partial T_{i}}{\partial \theta}
$$

where

$$
\nabla \cdot \mathbf{q}_{\perp i}=\frac{5}{2} \frac{p_{i} U_{T e}}{r} \frac{\partial}{\partial \theta} \ln \frac{n}{B^{2}}, \quad U_{T i}=\frac{1}{M_{i} \omega_{B i}} \frac{\partial T_{i}}{\partial r} .
$$

After integration over the angle $\theta$, we obtain from Eq. (30)

$$
\Gamma_{T i}=-\frac{2 n T_{i} v_{i} \cosh \eta}{M_{i} \omega_{B i}^{2}} \frac{\partial T_{i}}{\partial r}-\frac{5 c B_{s}}{4 \pi \varepsilon e_{i} R} \int_{0}^{2 \pi} d \theta \frac{p_{i}}{B^{2}} \frac{\partial T_{i}}{\partial \theta} .
$$

¿From this equation it also follows that the value for the heat flux depends explicitly on the ion poloidal velocity $V_{i}^{\theta}$.

\section{AMBIPOLARITY CONDITION}

The ambipolarity condition follows from the current continuity equation $\nabla \cdot \mathbf{j}=0$, i.e.,

$$
<j^{r}>=0 .
$$

It can be found from Eq. (41) that

$$
\begin{gathered}
j^{r} \sqrt{g}=\frac{c g_{33} B^{\zeta}}{B^{2}}\left\{-\left(\nabla p+\nabla \cdot \hat{\pi}+M_{i} n \frac{d \mathbf{V}_{i}}{d t}\right)_{\theta}+\right. \\
\left.+\frac{g_{22}}{q g_{33}}\left(\nabla \cdot \hat{\pi}+M_{i} n \frac{d \mathbf{V}_{i}}{d t}\right)_{\zeta}\right\}
\end{gathered}
$$

We take $\nabla \cdot \hat{\pi}$ in the form $[15]$

$$
\nabla \cdot \hat{\pi}=\frac{3}{2}\left\{[\mathbf{h}(\nabla \cdot \mathbf{h})+(\mathbf{h} \cdot \nabla) \mathbf{h}] \pi_{\|}+\mathbf{h}(\mathbf{h} \cdot \nabla) \pi_{\|}-\frac{1}{3} \nabla \pi_{\|}\right\} .
$$

Using the relations (taking into account that $\beta_{p} \ll 1$ )

$$
(\mathbf{h} \cdot \nabla) \mathbf{h}=-[\mathbf{h} \times[\nabla \times \mathbf{h}]] \approx-\left[\mathbf{h} \times\left[\nabla \frac{1}{B} \times \mathbf{B}\right]\right]=\nabla_{\perp} \ln B
$$

and

$$
\mathbf{h}(\nabla \cdot \mathbf{h})=-\mathbf{h}(\mathbf{h} \cdot \nabla) \ln B
$$

we find the components of this vector

$$
(\nabla \cdot \hat{\pi})_{\theta}=\frac{3}{2} \pi_{\|} \frac{\partial}{\partial \theta} \ln B-\frac{1}{2} \frac{\partial \pi_{\|}}{\partial \theta},
$$

$$
(\nabla \cdot \hat{\pi})_{\zeta}=\frac{3}{2} \frac{g_{33} B^{\zeta 2}}{q B^{2}}\left(-2 \pi_{\|} \frac{\partial}{\partial \theta} \ln B+\frac{\partial \pi_{\|}}{\partial \theta}\right)
$$


As follows from Eqs. (20), (41), we have used here the condition that $\nabla \times \mathbf{B}$ is proportional to the parameter $\beta_{p}$ which is small in solar loops. Using Eq. (33), we obtain

$$
M_{i} n\left(\frac{d \mathbf{V}_{i}}{d t}\right)_{\theta}=-M_{i} n V_{\|}^{2} \varepsilon^{*} \sin \theta
$$

The value $\partial p / \partial \theta$ can be derived from the longitudinal component of the summed electron and ion equation of motion, Eq. (38),

$$
M_{i} n \frac{d_{i} \mathbf{V}_{i}}{d t}=-\nabla p-\nabla \cdot \hat{\pi}_{i}+\frac{1}{c}[\mathbf{j} \times \mathbf{B}] .
$$

Multiplying Eq. (74) by h and using Eq. (71) - (73), we find

$$
\frac{\partial p}{\partial \theta}=-\frac{\partial \pi_{\|}}{\partial \theta}+M_{i} n V_{\|}^{2} \varepsilon^{*} \sin \theta
$$

where we have omitted the electron inertia and viscosity.

Neglecting the poloidal angle dependence of the electron temperature, we get from Eq. (75)

$$
\tilde{n}=-\frac{n \tilde{T}_{i}+\pi_{\|}}{T_{i}+T_{e}}-\alpha \varepsilon^{*} n \cos \theta
$$

where $\tilde{n}$ and $\tilde{T}_{i}$ are the oscillating, with the angle $\theta$, parts of the density and temperature.

Now the ambipolarity condition, Eq. (67), has the form

$$
\int_{0}^{2 \pi} d \theta\left[\frac{3}{2} \pi_{\|}\left(\frac{\partial}{\partial \theta} \ln B+\varepsilon^{*} A \sin 2 \theta\right)-\alpha \varepsilon^{*}\left(n \tilde{T}_{i}+\pi_{\|}\right) \sin \theta\right]=0
$$

To obtain this result we have used Eqs. (68), (71) - (73) and we omitted the last two terms inside brackets in Eq. (68).

As one can see from Eq. (77), we need to calculate the perturbed temperature and viscosity values. The temperature $\tilde{T}_{i}$ can be obtained from Eqs. (63) - (65), (76)

$$
\tilde{T}_{i}=-\frac{v_{i} M_{i} q^{2} R}{3.91} \frac{\varepsilon^{*}}{\varepsilon}\left\{5 U_{T i}\left[\left(1+\frac{\alpha}{2}\right) \sin \theta+\frac{A}{8} \sin 2 \theta\right]-\alpha U_{\theta i} \sin \theta\right\},
$$

where $U_{\theta i}=r V_{i}^{\theta}$.

We take the parallel viscosity tensor, $\pi_{\|}$, in the changed form [16]-[18], as for the problem under consideration, it is important to mention it, the viscosity tensor depends on the thermal fluxes $\mathbf{q}_{i}$. Using the usual viscosity expression [3], we are led to the conclusion that the equilibrium poloidal velocity of plasma rotation is zero. This problem was discussed in several occasions [19], [17]

$$
\pi_{\|}=-\frac{2}{3} \frac{p_{i}}{v_{i}}(0.96 \beta-0.59 \gamma)
$$

Here

$$
\begin{aligned}
& \beta=3\left\{\mathbf{h}(\mathbf{h} \cdot \nabla) \mathbf{V}_{i}+\frac{2}{5 p_{i}} \mathbf{h}(\mathbf{h} \cdot \nabla) \mathbf{q}_{i}-\frac{1}{3}\left(\nabla \cdot \mathbf{V}_{i}+\frac{2}{5 p_{i}} \nabla \cdot \mathbf{q}_{i}\right)\right\}, \\
& \gamma=-\frac{6}{5}\left\{\mathbf{h}(\mathbf{h} \cdot \nabla)\left(\mathbf{q}_{i}-\mathbf{q}_{i}^{*}\right)+\frac{1}{3}\left(\frac{M_{i}}{T_{i}} \mathbf{F}_{i} \cdot \mathbf{q}_{i}-\nabla \cdot\left(\mathbf{q}_{i}-\mathbf{q}_{i}^{*}\right)\right)\right\},
\end{aligned}
$$

$\mathbf{F}_{i}=\nabla p_{i} / M_{i} n$, and $\mathbf{q}_{i}^{*}=-0.27 \mathbf{q}_{\| i}$

To find the ion perturbed velocities in Eqs. (80), (81), oscil- 
lating with angle $\theta$, we proceed from the frozen-in condition

$$
\left[\nabla \times\left[\mathbf{V}_{i} \times \mathbf{B}\right]\right]=0
$$

and the continuity Eq. (36). From the $\zeta$-covariant component of Eq. (82), we derive

$$
\frac{\partial}{\partial \theta}\left[\sqrt{g}\left(V^{\theta} B^{\zeta}-V^{\zeta} B^{\theta}\right)\right]=0
$$

and, after using Eqs. (14), (15)

$$
\tilde{V}_{i}^{\zeta}=-q \tilde{V}_{i}^{\theta}
$$

¿From the continuity Eq. (36), which we rewrite in the form

$$
\frac{\partial}{\partial \theta}\left(n \sqrt{g} V_{i}^{\theta}\right)=0
$$

we have,

$$
\frac{\partial \tilde{V}_{i}^{\theta}}{\partial \theta}=-\frac{U_{\theta i}}{r} \frac{\partial}{\partial \theta} \ln (n \sqrt{g})
$$

Here, " $\tilde{V}$ " designates the parts that oscillate with the angle $\theta$. Now, we use the identities

$$
\begin{gathered}
\mathbf{h}(\mathbf{h} \cdot \nabla) \mathbf{V}_{i}=(\mathbf{h} \cdot \nabla) V_{i \|}-\mathbf{V}_{i}(\mathbf{h} \cdot \nabla) \mathbf{h}=(\mathbf{h} \cdot \nabla) V_{i \|}-\mathbf{V}_{i} \cdot \nabla_{\perp} \ln B, \\
\mathbf{h}(\mathbf{h} \cdot \nabla) \mathbf{q}_{i}=(\mathbf{h} \cdot \nabla) q_{i \|}-\mathbf{q}_{i} \cdot \nabla_{\perp} \ln B, \quad \tilde{V}_{i \|} \approx g_{22} h^{\theta} \tilde{V}^{\theta}+g_{33} h^{\zeta} \tilde{V}^{\zeta} .
\end{gathered}
$$

Further, using Eqs. (63) - (65), (76), (78) and (84) - (86), we find

$$
\begin{gathered}
\beta=\frac{3}{r}\left\{-U_{\theta i} \frac{\partial}{\partial \theta} \ln \left(\sqrt{g} n^{0.4} B\right)+U_{T i} \frac{\partial}{\partial \theta} \ln \frac{B}{n}\right\} \\
\gamma=-\frac{6}{5 r}\left\{\frac{38}{45} U_{\theta i} \frac{\partial}{\partial \theta} \ln n+U_{T i}\left(\frac{61}{18} \frac{\partial}{\partial \theta} \ln B-\frac{19}{9} \frac{\partial}{\partial \theta} \ln n\right)\right\} .
\end{gathered}
$$

Then, we finally derive the ambipolarity condition, using Eqs. (31), (78), (88) and (89)

$$
0.96\left(\frac{3}{2}+\alpha\right)\left\{U_{\theta i}(1+0.19 \alpha)+U_{T i}(1.83+1.52 \alpha)\right\}+
$$

$+\frac{b \alpha}{3}\left\{\alpha U_{\theta i}-5 U_{T i}\left(1+\frac{\alpha}{2}\right)\right\}+\frac{3}{8} 0.96 A^{2}\left(U_{\theta i}-1.83 U_{T i}\right)=0$,

where

$$
b=\frac{3}{2} \frac{M_{i} v_{i}^{2} q^{2} R^{2}}{3.91 T_{i}}
$$

is the collisionality parameter, which for the collisional plasma is much greater than one.

For circular tokamaks, Eq. (90) was obtained earlier [17]. For this case, it is necessary to put the elliptic parameter $A$ to be equal to zero. This expression gives us the possibility to find the ion ambipolar poloidal velocity $U_{\theta i}$.

\section{FLUXES ANALYSIS}

Now, we can derive the final expressions for the fluxes and analyze them. Let us rewrite Eq. (90) in the form

where

$$
U_{\theta i}=k U_{T i},
$$

We see, from Eq. (92), that it has the usual form for a colliWhen the longitudinal fluxes and the ellipticity are absent, i.e. 
the parameters $\alpha$ and $A$ are equal to zero, we have the well known result [17]

$$
U_{\theta i}=-1.83 U_{T i}
$$

which differs only slightly from the result of Hazeltine [19] (where, instead of the 1.83 value, the 2.1 value stands), ( as it depends on the method of the calculation). If the parameter $\alpha$ is to be allowed, we have the former result [17], where the property of the $k=k(\alpha)$ function has been studied. Now, we have taken into account the ellipticity in Eq. (93). In the well- known thermonuclear facilities, the value $A$ is around one. In this case, the ellipticity contribution is about 0.25 of the numerical coefficients in Eq. (93). But, it can be supposed that in the solar loops, in some cases, the parameter $A$ can be larger than one and the contribution of the ellipticity into $k$ is comparable with the other coefficients.

Even though the poloidal velocity $U_{\theta i}$ is interesting by itself, it is also important to know it for the evaluation of the transport fluxes in the plasma, let us show it. After using Eqs. (53) - (58), we obtain the expression for the particle fluxes

$$
\begin{gathered}
\Gamma_{n e}=\Gamma_{n i}=-\frac{c^{2} n}{\sigma_{\perp} B^{2}}\left\{\cosh \eta\left(p^{\prime}-\frac{3}{2} n T_{e}^{\prime}\right)+\right. \\
+q^{2}(2+\alpha) \frac{\varepsilon^{* 2}}{\varepsilon^{2}}\left[\frac{\sigma_{\perp}}{\sigma_{\|}}\left(1+\frac{0.71^{2}}{3.16} \frac{\sigma_{\|}}{\sigma_{\perp}}\right)\left(p^{\prime}+\frac{\alpha p}{2}\left(\frac{1}{r}+\kappa_{n}+2 \kappa_{V}\right)\right)-\right. \\
\left.\left.-\frac{5 \cdot 0.71}{2 \cdot 3.16} n T_{e}^{\prime}\left(1+\frac{\alpha}{2}\left(1-\frac{2}{5} \frac{U_{\theta e}}{U_{T e}}\right)\right)\right]\right\} .
\end{gathered}
$$

This equation includes previously received results, when the ellipticity and the particle longitudinal velocities are supposed to be small ( $\alpha=0$ and $\cosh \eta=1$ ) [21] or the $\alpha$ parameter is allowed [10]. We see from Eq. (95), that if one takes into account only the values $\cosh \eta$ and $\varepsilon^{* 2} / \varepsilon^{2}$, the role of the neoclassical effects is diminishing (as the factor $l_{1} / l_{2}$ ) and of the classical ones is increasing (as the factor $\left.\cosh \eta=\left(l_{2}^{2}+l_{1}^{2}\right) / 2 l_{1} l_{2}\right)$. But we should take into account the ratio $U_{\theta e} / U_{T e}$ additionally, where

$$
U_{\theta e}=U_{\theta i}+\frac{\varepsilon}{q} \frac{j_{\|}}{e_{e} n}+U_{p}, \quad U_{p}=\frac{p^{\prime}}{M_{e} n \omega_{B e}} .
$$

The ellipticity can also affect the particle fluxes through the dependence $U_{\theta e}$ on $U_{\theta i}$.

Ion heat flux can be derived from Eqs. (63) - (66)

$$
\begin{gathered}
\Gamma_{T i}=-\frac{2 n T_{i} \vee_{i}}{M_{i} \omega_{B i}^{2}} T_{i}^{\prime}\left\{\cosh \eta+1.6 q^{2} \frac{\varepsilon^{* 2}}{\varepsilon^{2}}\left[\left(1+\frac{\alpha}{2}\right) \times\right.\right. \\
\left.\left.\times\left(1+\frac{\alpha}{2}\left(1-\frac{2}{5} \frac{U_{\theta i}}{U_{T i}}\right)\right)+\frac{A^{2}}{16}\right]\right\} .
\end{gathered}
$$

We obtain the previously known results, if we put here $\eta=$ $\alpha=A=0$ [14] or $\eta=A=0$ [8]. [9]. The ellipticity included in Eq. (97) increases the role of the classical effects and diminishes the role of the neoclassical one, as in the collisional case, if only the $\eta$ and $\varepsilon^{*}$ effects are taken into account. The toroidal flow (the parameter $\alpha$ ) can also decrease the role of the neoclassical term in the ion radial heat flux in some range of the values of the collisional parameter $b$ [8], [9].

\section{SOME EVALUATIONS}

Now, we make some evaluations. There are some kinds of the solar loops that exist on the Sun [1], [2]. The solar loop parameters can be found in a wide range of values: the lengths $L$ are from $5 \times 10^{3} \mathrm{~km}$ to $7 \times 10^{5} \mathrm{~km}$, the temperatures $T$ are from $10^{4} \mathrm{~K}$ to $4 \times 10^{7} \mathrm{~K}$, the densities $n$ between $2 \times$ $10^{14} \mathrm{~m}^{-3}$ and $10^{18} \mathrm{~m}^{-3}$, the toroidal magnetic fields $B_{\zeta_{p}}$ are between some Gauss to some $\mathrm{kG}$ and poloidal magnetic fields $B_{\theta p}$ reach $10 \mathrm{G}$. Siphonic flow velocities can reach $200 \mathrm{~km} / \mathrm{s}$, but they are usually about $20-30 \mathrm{~km} / \mathrm{s}$, i.e. they are smaller or the same order of magnitude of the plasma sound speed, Eq. (53).

With these parameters, the collision frequency of the $k$-kind particles, $v_{k}$, satisfy the condition $T_{k} / M_{k} q R<v_{k}<\omega_{B k}$; the particle mean free path is usually smaller than the loop crosssection radius $r$; the parameter $\beta_{p}=8 \pi p / B^{2}$ satisfies the condition $\beta_{p} \ll 1$. Then, we can use the collisional neoclassical model [12] for the transverse transport calculation.

The pressure and temperature in the core of a loop are always lower than in the surrounding sheath, but they can be either larger or smaller than the ambient coronal values. We see from Eqs. (95), (97) that the particle and heat fluxes are on the "classical" level, maybe within a factor of an order of the magnitude if the parameters $\alpha$ and $A$ satisfy the reasonable conditions $\alpha \lesssim 1$ and $A \lesssim 1$. They can be essential when the temperature and density profiles are sharp, e.g. on the border between the sheath and the core of the solar loops.

The plasma poloidal velocity, Eqs. (92), (93), can reach large values in the solar loops with the temperature sharpened 
profile. For example, for the temperature $T=10^{6}-10^{7} \mathrm{~K}$, the magnetic field $B=1-10 \mathrm{G}$, the radial gradient $\partial T / \partial r \approx$ $T / \Delta r$, where $\Delta r \approx 1 \mathrm{~km}$, we have

$$
U_{\theta i} \geq 10-10^{6} \mathrm{~cm} / \mathrm{s}=10 \mathrm{~km} / \mathrm{s},
$$

i.e.. it can be on the level of the siphonic flow and can be measured experimentally. This measuring gives us the possibility to have additional connections between the plasma macroscopic parameters, that is very important for the solar loop diagnostic. For example, at the some siphonic velocity and ellipticity values, the poloidal velocity Eqs. (92), (93) changes sign, and can be also measured.

\section{CONCLUSION}

The general theory of the neoclassical transport in toroidal configurations, well-developed in connection with the thermonuclear investigations, is applied to explain the transport processes in some kinds of toroidal solar loops. It is supposed, that they have the noncircular cross-section, and there are the particles longitudinal flows along the solar loops. The plasma is also supposed to be collisional and with two components, ions and electrons.

The poloidal velocity, radial fluxes and the ion heat flux of the particles were derived in this article. The dependence of these values on the ellipticity parameters $\eta=\ln \left(l_{2} / l_{1}\right)$ and $A=\varepsilon^{*}(\exp (2 \eta)-1) / q^{2}$, where $l_{2}$ and $l_{1}$ are the large and the minor half-axis of the ellipse, and on the parameter
$\alpha=V_{\|}^{2} / c_{s}^{2}$, connected with the particle longitudinal (along the magnetic field lines) velocities $V_{\|}$and the sound velocity $c_{s}$, was found.

It is shown that, in the toroidal configurations, with the elliptic transverse cross-section in the plasma transverse transport processes, the role of the classical effects increases as $\cosh \eta$ and of the neoclassical effects decreases as $\exp (-\eta)$, at a not too large parameter $A$. Although the ellipticity usually does not give a substantial contribution to the expressions for the transport fluxes, we believe that it is useful to take the ellipticity into account methodically for future investigations. It is noted that, as in the circular cross-section tokamak, the neoclassical ion heat flux decreased at some range of the parameter $\alpha$ and the collisional parameter $b=$ $3 M_{i} v_{i}^{2} q^{2} R^{2} /\left(2 \cdot 3.91 T_{i}\right)$.

It is also confirmed that for these configurations the formula for the ion poloidal velocity $U_{\theta i}=k U_{T i}$ is the same as the one for a circular tokamak. It is possible that the poloidal velocity can be measured in the solar loops by means of the Doppler shift.

We suppose that such the approach (together with others theories), in spite of the fundamental differences between solar loops and thermonuclear facilities, can help to explain the transport processes in solar loops, which form is similar to toroidal configurations.

Acknowledgements. This work was partially supported by the Research Foundation of the State of São Paulo (FAPESP) and by the National Council of Scientific and Technological Development (CNPq).
[1] G. W. Pneuman and F. Q. Orral, Physics of the Sun, (D. Reidel Publ. Co., Dordrecht, Holland, 1980), Vol. 2, p. 71.

[2] E. Priest, Solar Magnetohydrodynamics, (D. Reidel Publ. Co., Dordrecht, Holland, 1980).

[3] S. I. Braginskii, in Reviews of Plasma Physics, edited by M.A.Leontovich (Consultants Bureau, New York, 1965), Vol. 1, p. 205.

[4] F. Wagner and U. Strouth, Plasma Phys. Control. Fusion 34, 1803 (1992)

[5] F. L. Hinton and R. D. Hazeltine, Rev. Mod. Phys. 48, 239 (1976).

[6] P. A. Sturrock, T. E. Holzer, D. M. Mihalas, and R. K. Ulrich, Physics of the Sun, (D. Reidel Publ. Co., Dordrecht, Holland, 1986).

[7] S. K. Wong and K. H. Burrel, Phys. Fluids 25, 1863 (1982).

[8] S. K. Wong and F. L. Hinton, Phys. Rev. Lett. 52, 827 (1984).

[9] V. S. Tsypin, Sov. J. Plasma Phys. 14, 466 (1987).

[10] V. S. Tsypin, Sov. J. Plasma Phys. 11, 201 (1985).

[11] V. D. Shafranov and L. S. Solovev, in Reviews of Plasma
Physics, edited by M.A. Leontovich (Consultants Bureau, New York, 1969), Vol. 5, p. 3.

[12] D. Pfirsch and A. Schlüter, Max Plank Institute, Report MPI/PA/7/62 (1962).

[13] R. D. Hazeltine and F. L. Hinton, Phys. Fluids 16, 1883 (1973).

[14] V. D. Shafranov, Sov. Atom. Energy 19, 1008 (1965).

[15] G. F. Chew, M. L. Goldberger, and F. E. Low, Proc. Royal Soc. A236, 112 (1956).

[16] A. B. Mikhailovskii and V. S. Tsypin, Plasma Physics 13, 785 (1971).

[17] A. B. Mikhailovskii and V. S. Tsypin, Sov. Phys. JETP 56, 75 (1982).

[18] A. B. Mikhailovskii and V. S. Tsypin, Beiträge Plasma Physik 24, 335 (1984)

[19] R. D. Hazeltine, Phys. Fluids 17, 961 (1974).

[20] F. L. Hinton and M. N. Rosenbluth, Phys. Fluids 16, 836 (1973).

[21] V. D. Shafranov, J. W. Connor, and C. J. H. Watson, Sov. J. Plasma Phys. 2, 99 (1976). 